\title{
Le piercing lingual et ses complications : implications militaires
}

\author{
Mathieu Gunepin ${ }^{1, \star}$, Florence Derache ${ }^{2}$, Emmanuel Blin $^{3}$, Éric Morell ${ }^{4}$ \\ 1 Secteur dentaire Inter-armées, Draguignan, France \\ 2 Service de Chirurgie maxillo-faciale et Stomatologie, HIA Sainte Anne, Toulon, France \\ 3 Service de Cardiologie et Médecine vasculaire, HIA Bégin, Saint Mandé, France \\ ${ }^{4}$ Service d'Anesthésie-Réanimation, HIA Bégin, Saint Mandé, France
}

(Reçu le 6 juillet 2009, accepté le 27 août 2009)

Mots clés :

piercing lingual /

complications /

odontologie /

armée

Key words:

tongue piercing /

complications /

odontology /

army

\begin{abstract}
Résumé - Avec plus de 100000 actes par an en France, le piercing en général et le piercing lingual en particulier sont désormais un phénomène de société. Avec des complications multiples, très fréquentes (près d'un porteur de piercing lingual sur 2 a au moins une dent fracturée du fait du piercing) et parfois létales, le piercing lingual est devenu un problème de santé publique.

La prise en compte de ces complications se limite actuellement à la maîtrise de la transmission des agents infectieux lors du perçage. Ceci semble extrêmement réducteur comparé aux nombreuses conséquences délétères de la pose, puis du port du piercing lingual qui ont incité plusieurs sociétés savantes civiles à prendre position contre le perçage de la langue.

Dans ce contexte l'armée française, en s'appuyant sur des faits scientifiques avérés, doit pouvoir répondre à une pratique à même de remettre en question la capacité opérationnelle de ses forces.
\end{abstract}

\begin{abstract}
Tongue piercing and its adverse effects: military implications. In western populations, the insertion of metal objects into intra-oral pierced sites is growing in popularity. This practice is considered by consumers as totally trivial and most of them have absolutely no idea of the consequences of tongue piercing. In fact, complications are frequent and can appear due to the intervention and/or during the carrying of the piercing.

Firstly, during the intervention can appear infectious and/or non infectious complications. Infections, viral or bacterial, as consequences of the piercing are very frequent (in 10 to $30 \%$ of piercing). Some of these complications are life threatening. Non infectious complications are pain, swelling, bleeding, lingual nerve damage...

Secondly, during carrying of tongue piercing can appear odontological and/or non odontological complications. Non odontological complications are secondary infectious, swallowed or inhaled jewellery, pathological healing, allergic reactions, hypersalivation, speech impediment... Odontological complications are very frequent with deleterious consequences: cracking or chipping of teeth, abrasions, gingival recession or loss of periodontal attachment...

The growing in popularity of tongue piercing is also a reality in the french army. The analysis of cases reports and international (civilian and military) recommendations make us consider tongue piercing as a factor of decreasing operational capability of military troops. For this reason we recommend the absolute interdiction of tongue piercing during military operations.
\end{abstract}

Le piercing, terminologie couramment utilisée en France pour désigner le terme anglais body piercing, regroupe diverses pratiques de modification corporelle qui se caractérisent par une effraction de la barrière cutanée (le plus souvent à l'aide d'aiguilles) avec mise en place durable d'un objet

\footnotetext{
^Correspondance : mgunepin@yahoo.fr
}

(le plus souvent métallique) extérieurement et partiellement visible [1].

Au cours des dernières décennies, le piercing est devenu de plus en plus courant au sein des populations occidentales [2-4]. Cet engouement s'est traduit par une augmentation de la prévalence des complications liées à la pose et au port de ces bijoux corporels. Il s'agit désormais d'un vrai problème de santé publique. 
Si le perçage de divers sites anatomiques est une pratique qui remonte à plusieurs milliers d'années, les motivations aboutissant à cet acte ont évolué au fil des temps et des civilisations : décoration, rite de passage, motifs religieux, pratiques sexuelles [2-7]. Dans les sociétés occidentales, le piercing s'est communément limité pendant des années aux lobes des oreilles, les autres sites étant alors considérés comme non-traditionnels et utilisés par des populations marginales $[2,3,6]$. Ceci n'est plus vrai aujourd'hui et parmi les localisations préférentielles de perçage on trouve le piercing de la langue qui sera étudié plus particulièrement ici.

L'objectif de ce travail est double : l'un de santé publique et l'autre médico-militaire. D'un point de vue de santé publique, même si ces pratiques sont difficiles à admettre sur le plan médical (nombreux effets indésirables avec un retentissement local ou sur l'organisme entier), tout porte à croire que le nombre de patients avec un ou plusieurs piercings dans la cavité buccale va augmenter dans les prochaines années $[2,8,9]$. Or, les médecins et les chirurgiens dentistes sont au premier plan pour informer les patients des risques et dépister les complications. C'est pourquoi on donnera des informations aux professionnels de santé, notamment militaires, concernant le port de plus en plus fréquent de bijoux corporels. La connaissance de la terminologie appropriée, des techniques de pose, etc. permettra d'engager avec les patients un dialogue dégagé de tout a priori stigmatisant les individus «percés » $[10,11]$. D'un point de vue médico-militaire, la question de l'impact opérationnel du piercing lingual doit être posée. Le piercing lingual est d'ores et déjà interdit au sein des armées américaine, israélienne et australienne; on verra ce qui a justifié ces prises de position et en quoi elles seraient transposables pour l'armée française.

\section{Historique}

\section{Piercings d'hier}

Les rites s'apparentant à la perforation du corps semblent avoir pris naissance à la genèse de l'humanité. Le besoin de communiquer avec ses congénères a poussé l'Homme à la réalisation de peintures, de dessins, d'ornements et de modifications corporelles avant même l'apparition d'un langage élaboré. Les plus anciennes parures corporelles furent découvertes au Tchad, elles datent de l'époque néolithique. Il s'agit de labrets de pierre, objets servant à orner la lèvre inférieure en la transperçant de part en part. Des boucles narinaires et des pendentifs de cloison nasale datant de l'ère précolombienne ont été retrouvés en Amérique du Sud, ainsi que des bijoux d'oreille âgés de plus de 5000 ans ayant appartenu à un pharaon. Les premiers piercings linguaux sont retrouvés dans les civilisations aztèques et mayas [10]. Pour les Shamans et les Grands prêtres, se percer la langue faisait partie d'un rituel pour communiquer avec leurs Dieux [12].

\section{Piercings d'aujourd'hui}

C'est dans les années 1970 sur la côte ouest américaine qu'apparaissent les techniques modernes de piercing.
La première boutique de piercing a ouvert ses portes en 1975 à Los Angeles avec une succursale à Paris en 1995. Dès lors le rite d'inclusion ancestral s'est subitement transformé en rite d'exclusion pour des individus considérant que la seule manière d'échapper au conformisme ambiant consiste à individualiser son corps en le modifiant. Il s'agit au départ de pratiques émanant de populations marginales comme les grunges, les gothiques, les milieux homosexuels et skins, les héritiers du punk et les sadomasochistes. La démocratisation de ces pratiques se fait dans les années 1990 lorsque le public fasciné porte aux nues un sadomasochisme considéré comme le comble du chic.

Malgré cette « acceptation générale » du phénomène piercing, une connotation négative reste liée à ces pratiques. Ainsi une enquête menée par la société Vault [13], spécialisée dans les questions d'emplois aux États-Unis, indique que $85 \%$ des individus sondés pensent que le tatouage et le piercing ont un impact négatif dans la recherche d'un emploi. De plus $53 \%$ des employés cachent leurs piercings lorsqu'ils sont au travail par peur d'être stigmatisés et rejetés. Le fait de pouvoir être perçus par la société comme ayant un comportement déviant explique certainement le fait que la plupart des patients retirent leur piercing avant d'aller chez leur chirurgien dentiste [14].

\section{Motivations du « percé »}

Les préjugés sont grands et la méconnaissance du contexte psychosocial et des motivations des «percés » amène à considérer cette pratique comme pathologique. La plus grande partie de la littérature publiée jusqu'à il y a peu de temps sur le sujet était imprégnée d'un fond de discrimination, la présence de modifications corporelles comme les tatouages ou le piercing étant associée à des comportements psychopathologiques ou antisociaux [15]. On parlait «d'estropier le corps », « d'atteinte à l'intégrité physique due à la mode », «de destruction volontaire du propre tissu corporel», etc [15]. Dans la société actuelle où les piercings se trouvent dans toutes les couches sociales, le recours à des interprétations discriminatoires et la considération de ces pratiques comme exclusivement pathologiques ne sont plus permis. Il est cependant certain que l'association « blessure volontaire - perversion » se conçoit facilement. Mais le piercing n'a rien à voir avec un plaisir pathologique de la douleur; la douleur qui accompagne le piercing n'est qu'un effet secondaire [15].

Pourquoi se faire percer? Une réponse pourrait être qu'il existe autant de motivations que d'adeptes du piercing : soucis de rébellion envers la société, contre l'autorité parentale ; souhait d'individualiser son corps vis-à-vis de la "masse »; phénomène d'identification à des personnages connus du monde du cinéma, de la chanson...; quête de réappropriation du corps passant par la souffrance de celui-ci; recherche de vertus sexuelles (augmentation du plaisir sexuel pour le ou la partenaire [16]); possibilité de bénéfices spirituels [17]; raisons esthétiques [18]; faire montre de courage et d'audace ; 
désir d'appartenance à une communauté, un groupe ; pression des pairs; etc.

Les motivations sont multiples, c'est pourquoi l'association piercing - érotisme, sadomasochisme et fétichisme n'est probablement pas applicable à la situation actuelle et ne peut être maintenue comme seule explication du phénomène. Dans une enquête réalisée auprès des lecteurs de la revue Body Art (revue spécialisée sur le piercing, le tatouage et autres pratiques de modifications corporelles à visée esthétique), plus de $80 \%$ des sujets interrogés déclaraient n'être «ni sadomasochistes, ni fétichistes, ni exhibitionnistes, ni narcissiques » [19].

\section{Données épidemiologiques}

Aujourd'hui les sites de piercing les plus populaires sont les oreilles, les sourcils, les lèvres, le nez, les tétons, le nombril, le pénis, le scrotum, le clitoris et la langue $[9,20]$. Une étude SOFRES réalisée en 2003 sur un échantillon de 500 personnes représentatif de la population âgée de 15 à 24 ans montre que $43 \%$ des sujets sondés envisagent de se faire poser un piercing (59\% de filles, $27 \%$ de garçons) [12]. En 2005 , on comptait 900 perceurs professionnels en France et 5 millions de personnes portant au moins un piercing avec une répartition par sexe entre 2003 et 2005 de $72 \%$ de femmes pour $28 \%$ d'hommes. En France, plus de 100000 actes de piercing seraient pratiqués chaque année sur une clientèle variée. Cette engouement progressif peut être mesuré au nombre de studios de piercing en France : 4 en 1980, 50 en 1990, 100 en 1995, 400 en 2000 et plus de 1000 en 2005 [12].

Le succès du piercing s'explique notamment par le caractère réversible de la pose de ces bijoux corporels [21]. En ce qui concerne la cavité buccale, la lèvre a longtemps été le site de perçage le plus commun, mais la langue est en train de s'imposer comme localisation préférentielle [16]. Les piercings buccaux concernent essentiellement une population jeune, de 18 à 30 ans [22] et plus particulièrement les femmes [23].

En ce qui concerne la population américaine, l'enquête de la société Vault [7] s'intéressant au lien entre piercing et marché du travail montre que $20 \%$ des sujets sondés (représentatifs de la population américaine) ont un ou plusieurs piercings ( $13 \%$ un, $5 \%$ deux et $2 \%$ trois ou plus) ; $7 \%$ de ces piercings sont localisés dans la langue. La langue est le site de perçage le plus commun en ce qui concerne la sphère buccale [24].

\section{Conditions et techniques de pose du piercing lingual}

La pose du piercing lingual se pratique sans anesthésie. L'opérateur n'appartient bien évidemment pas au corps médical et il exerce en autodidacte. Dans le meilleur des cas, il officie dans une échoppe de piercing et se conforme à la réglementation en vigueur (code de la santé publique). Il faut savoir qu'il existe également des « coiffeurs-perceurs » travaillant dans des salons de coiffure et même des «perceurs ambulants » qui transportent leur matériel sur les marchés, dans les fêtes (rave parties, etc.) ou dans les boîtes de nuit. Enfin le piercing est parfois réalisé par la personne elle-même, mais la fréquence de l'auto-piercing est impossible à connaître à ce jour en France.

Dans la langue, la localisation dorso-ventrale, centrale, est la plus répandue. On évoquera que ce type de piercing. Il faut cependant savoir que d'autres localisations existent : dorsolatérale, décentrée ou encore une localisation transversale, traversant la langue horizontalement. Certains n'hésitent pas, d'ailleurs, à multiplier toutes ces localisations.

Dans le cas du perçage de la langue, celle-ci est marquée par un point au stylo sur la ligne médiane en avant du frein [9]. Tenue par une pince, la langue est alors percée dans le sens ventro-dorsal au moyen d'une aiguille de large diamètre (calibre 14 ou 16 Gauge) [16]. Une canule en plastique souple peut ensuite être insérée autour de l'aiguille. Lorsque cette canule a traversé la langue de part en part, l'aiguille est retirée et le bras métallique du piercing introduit à sa place. La canule est finalement retirée à son tour. La partie femelle, de forme sphérique, peut alors être vissée sur la partie mâle et serrée à la main ou avec deux pinces. Entre les deux sphères, le bras de ce premier piercing provisoire doit avoir une longueur approximative de 18 à $20 \mathrm{~mm}$ (bijou dit de pose), faute de quoi l'objet risque de pénétrer dans l'épaisseur de la langue [25]. Approximativement deux semaines après l'intervention, le bras du piercing de $18 \mathrm{~mm}$ est retiré et un bijou permanent définitif est mis en place (bijou dit fantaisie). Sa longueur varie de 12 à $15 \mathrm{~mm}$. Au total, en l'absence de complications, la guérison prendra trois à six semaines selon les auteurs $[11,26]$. Cette cicatrisation rapide est liée à la nature de la vascularisation de la langue [14].

\section{Bijoux \\ Matériaux}

Les matériaux utilisés pour les bijoux sont multiples : acier, or, titane, niobium, acrylique, téflon, argent... Celui que l'on rencontre le plus est l'acrylique car c'est dans cette gamme que l'on trouve les bijoux les moins chers. L'acier est aussi très répandu pour les mêmes raisons. Par contre le titane est très apprécié pour ses qualités antiallergiques [27]. L'acier utilisé est un acier inoxydable chirurgical $316 \mathrm{~L}$ ou 316 LVM, conçu pour les bijoux corporels. Le titane de type TI6AL4VF136 est apprécié car léger, confortable et résistant mais par contre aussi plus onéreux. Il en est de même pour le niobium, métal brillant gris prenant une couleur bleutée quand il est exposé à l'air, très bien toléré et assez recherché pour sa couleur. L'acrylique ou polyméthylmétacrylate (PMMA) a une couleur vive et permet à peu près toutes les formes imaginables. Le téflon est aussi très bien toléré mais il est un peu plus cher. L'argent a été utilisé malgré ses problèmes d'oxydation ainsi que tous les matériaux « naturels » : bois, corne, ivoire, verre, os... 


\section{Terminologie}

Un véritable lexique serait nécessaire pour décrire la panoplie de possibilités offertes. Il est important pour le praticien d'utiliser les termes exacts lorsqu'il entame un dialogue à ce sujet avec son patient. Le barbell est la forme la plus classique : c'est une barre droite dont les extrémités sont agrémentées d'une boule ou d'un pic ou spike. Une extrémité est fixe, l'autre est à visser ou clipper. Le labret est la forme qui se rapproche le plus du barbell; c'est aussi une barre droite mais l'extrémité fixe est constituée par un disque plat. L'autre extrémité, à visser ou à clipper comme pour le barbell, peut avoir une multitude de forme : boule, pic ou encore étoile, cube... Il s'appelle labret s'il est situé dans la langue ou la lèvre inférieure. Il prend le nom de mouche, madonna ou médusa en fonction de ses autres localisations. L'anneau est fermé par une ou deux boules. On le retrouve dorso-latéralement sur la langue. Le banana bell ressemble au barbell mais sa tige est courbée (en forme de banane) et terminée à chaque extrémité par une boule ou un pic. Il peut être utilisé dans la langue.

\section{Complications liées au piercing lingual}

10 à $30 \%$ des poses de piercings comportent des complications. Dans le cas des piercings linguaux, ces dernières sont nombreuses et bien identifiées. On peut les regrouper en deux catégories :

- les complications liées à la pose du piercing ;

- les complications liées au port du piercing.

\section{Complications liées à la pose du piercing}

\section{Les complications infectieuses}

Une étude française indique qu'entre 10 et $20 \%$ des piercings se compliquent d'une infection locale [1] ; ce pourcentage pourrait atteindre $30 \%$ selon le groupe français d'étude et de recherche sur le piercing [28]. Ces pourcentages correspondent à ceux constatés aux États-Unis [29], Yagoda Shagam signale $25 \%$ de complications infectieuses [30]. Parmi les bactéries en cause, le staphylocoque doré, les streptocoques du groupe $A$ et le bacille pyocyanique sont les plus fréquemment trouvés. Ils peuvent donner lieu à des complications graves mettant en jeu le pronostic vital. Un rapport de la commission européenne publié en 2003 va plus loin car il indique que plus de la moitié des piercings peuvent entraîner des infections aiguës [31]. Entre fin 2002 et mi-2003, deux cas de décès consécutifs à une infection imputable à un piercing ont été rapportés en Europe.

On peut schématiquement distinguer deux types de complications infectieuses selon leur origine bactérienne ou virale. Pour les infections bactériennes, il est difficile de distinguer les infections liées à l'inoculation d'agents infectieux au cours du geste du piercing (matériel contaminé ou procédure inadéquate) de celles acquises secondairement (manipulation du bijou et/ou soins locaux inadaptés pendant la phase de cicatrisation). La survenue d'endocardites est également décrite (Haemophilus aphrophilus endocarditis [32], Nesseiria endocarditis [33], Streptococcus viridans endocarditis [34]...). Il est certain que le fait de transpercer la muqueuse et les muscles peut produire des infections locales consécutives à l'introduction de la microflore buccale dans les muscles $[35,36]$. Du fait de la vascularisation importante de ces muscles, il y a un risque secondaire de formation d'abcès à distance ou de septicité avec notamment un TSS (Toxic Shock Syndrom) qui est potentiellement fatal. Dans certains cas, la pose d'un piercing peut engager le pronostic vital [37]. Ainsi une jeune femme de 25 ans a du être hospitalisée pour une angine de Ludwig avec cellulite submandibulaire et sublinguale secondaire à la pose d'un piercing lingual 4 jours plus tôt [38]. 3 drains extraoraux furent placés sur la patiente intubée et ventilée avec une antibiothérapie IV. Au total la patiente a été ventilée artificiellement pendant 108 heures. Un cas d'abcès au cerveau a également été reporté par l'université de Yale $[39,40]$ chez une femme de 22 ans. Du fait de la douleur, de l'œdème et d'un écoulement purulent, la patiente avait retiré le piercing 3 jours après sa pose. La disparition des symptômes inflammatoires a été quasi immédiate, par contre la patiente a présenté 4 semaines plus tard des nausées, des vomissements, des vertiges et des céphalées. Elle a du être hospitalisée pendant 6 semaines. Des cas de tétanos ont également été décrits, et le piercing identifié comme le nouveau clou rouillé de ce début de millénaire [41].

Les infections virales sont plus faciles à rapporter à un geste de piercing récent, lorsqu'elles sont aiguës, pour peu que l'on interroge soigneusement les patients dans ce sens. En effet, le lien de causalité n'est pas forcément soulevé spontanément par le patient lui-même. Lorsque les infections virales sont asymptomatiques, l'imputation à un geste antérieur de piercing est beaucoup plus délicate et nécessite une enquête plus approfondie (recherche de tests sérologiques antérieurs, interrogatoire précis du patient pour la recherche d'autres facteurs de risque). On peut d'ailleurs présumer que les infections virales secondaires au piercing sont de ce fait vraisemblablement sous-évaluées. Plusieurs études épidémiologiques dans différentes populations ont retrouvé le piercing comme facteur de risque d'infection à VHC (virus de l'hépatite C) au même titre que l'usage de drogue par voie intraveineuse [4244]. Ces facteurs de risque ont un point commun, l'association d'une effraction cutanée et de l'utilisation d'aiguilles. Le risque de transmission virale par le piercing est apparu suffisamment important pour justifier dès 1998, par décision du Conseil de l'Union européenne, l'exclusion de tout donneur de sang ayant eu un piercing dans l'année précédant le don [45]. Au-delà du risque de transmission du VHC, le piercing lingual peut également être à l'origine d'une contamination par le virus de l'hépatite $B$, le virus d'Epstein-Barr ou différents Candida [46]. La possible transmission du virus HIV type 1 a été décrite par Pugatch et al. [47] : le patient ayant présenté une séroconversion à la suite de multiples actes de piercing. 
Des cas de transmission du virus herpes simplex sont bien documentés avec notamment le décès d'une femme contaminée par ce virus lors de la pose d'un piercing lingual : la maladie a progressé vers une hépatite fulminante qui a conduit au décès de la patiente [48].

\section{Complications non infectieuses}

Les deux complications principales liées à la pose d'un piercing lingual sont la douleur et l'œdème de la langue [20, 49]. En l'absence de toute anesthésie, la douleur est évidemment inévitable et parfois très intense. Elle est aussi fonction de l'expérience et de l'habileté du perceur. L'œdème est une constante lors de toute pose de piercing lingual du fait de la vascularisation importante de cette zone. Dans certains cas, rares, l'œdème peut causer l'obstruction des voies aériennes et digestives allant jusqu'à mettre en jeu le pronostic vital du patient et rendant nécessaire une intubation $[50,51]$. Dans la description de la technique de perçage et de mise en place du piercing, on a indiqué l'importance de la mise en place initiale d'un bijou de pose d'une longueur de 18 à $20 \mathrm{~mm}$ afin de prendre en compte l'œdème de la langue. Dans certains cas, même une longueur de $20 \mathrm{~mm}$ ne suffit pas [46], puisque deux semaines après la pose d'un piercing, la sphère se trouvant sur la face ventrale de la langue peut se retrouver totalement enchâssée à l'intérieur de la langue, nécessitant alors un geste chirurgical local. Cette complication est reportée de manière récurrente dans la littérature internationale malgré le respect des « recommandations professionnelles» $[52,53]$. Sa survenue serait en partie due à des parafonctions mises en place par le patient comme en exerçant une pression sur le piercing non cicatrisé avec les incisives notamment.

Un saignement prolongé peut également être constaté. Un cas de collapsus hypotensif nécessitant une hospitalisation d'urgence a même été reporté [50]. Glenn Rosivack et al. [54] indiquent_chez un patient de 15 ans un cas de saignement prolongé après à la pose d'un piercing. Le patient a alors retiré le piercing sans qu'aucun signe de cicatrisation ne survienne. Il est important de préciser que ce patient ne prenait aucun médicament et ne présentait aucune pathologie notamment du type trouble de l'hémostase. Le patient a du être admis aux urgences pédiatriques pour stopper l'hémorragie. Les hémorragies liées aux piercings linguaux restent cependant peu fréquentes, ces derniers étant généralement posés sur la ligne médiane de la langue et les vaisseaux sanguins se trouvant dans les parties latérales [55].

Des lésions nerveuses peuvent également survenir par atteinte des nerfs glossopharyngiens, hypoglosse, trigéminal ou facial (pour les piercings péri-buccaux). En ce qui concerne le piercing de la langue, les lésions sont plus fréquentes lors des perçages dorso-latéraux que latéraux. Les conséquences peuvent être, en fonction du nerf lésé, sensorielles (goût) ou motrices.

À noter pour terminer le cas d'un patient qui s'est retrouvé avec une langue bifide suite à son perçage [56] avec des conséquences psychologiques considérables pour le patient.

\section{Complications liées au port du piercing}

\section{Complications non odontologiques}

Même en cas d'absence d'infection directement liée à l'acte de percer, le risque infectieux perdure [57]. Ceci est dû au fait que, d'après la littérature [58], il n'y a pas de véritable guérison du lumen, ce qui place le patient à haut risque d'infection croisée même après la période de cicatrisation de 4 semaines.

La possibilité d'une ingestion, voire d'une inhalation de tout ou partie du piercing est également à prendre en considération $[46,59,60]$. La manipulation fréquente du piercing, notamment pour son nettoyage, engendre un risque d'une mise en place incorrecte du bijou qui peut quitter son emplacement. Les risques pour le patient sont alors extrêmement importants. Il faut rappeler que les composantes d'un piercing sont le plus souvent vissées à la main et que le patient doit en contrôler le serrage aussi souvent que nécessaire. Reyna et al. rapportent le cas d'un jeune homme de 17 ans ayant inhalé son piercing lingual, ce qui a nécessité une thoracotomie pour récupérer la pièce métallique [61].

Les cicatrisations pathologiques notamment chéloïdes sont des complications plus tardives du piercing [62], dont l'incidence n'est pas bien connue [63].

Un cas de courant galvanique a été reporté du fait du contact entre la partie du piercing en acier inoxydable et un volumineux amalgame dentaire [64].

L'hypersalivation [55] et la formation de calcul à la surface du barbell ont été observés, ceci ayant pour conséquence des interférences avec l'élocution, la déglutition et la mastication [65-68].

Les piercings peuvent également être source d'exposition à des allergènes comme le cobalt et le nickel [69]. L'acier chirurgical 316LVM contient une petite quantité de nickel qui peut provoquer une allergie. Une enquête du réseau d'allergovigilance français en 2007 montre que sur 138 allergologues ayant répondu à deux questionnaires, 7,9\% ont constaté des allergies aux piercings [70]. Bien qu'il existe aujourd'hui un contrôle rigoureux des matériaux utilisés dans le cadre du perçage, les dermatologues ont constaté une augmentation de l'incidence des allergies aux métaux (surtout au nickel et au cobalt) en corrélation positive avec l'augmentation de l'incidence du piercing dans la population générale [71]. De plus, le recours au piercing «forain » représente un risque de mise en place de bijoux non-conformes à la réglementation en vigueur. Les symptômes sont : un œdème de la muqueuse autour du bijou avec un érythème persistant, parfois un écoulement liquide transparent et de l'urticaire. Le traitement nécessite l'ablation du bijou.

À noter enfin une névralgie atypique associée à un piercing lingual [35] chez une jeune femme de 18 ans. La patiente présentait une névralgie faciale un mois après la pose d'un piercing lingual avec des douleurs paroxysmiques dans les régions du V2 et V3 à droite, suivies d'une hypoesthésie 30 secondes plus tard et ce 20 à 30 fois par jour. Le traitement par carbamazépine s'est révélé inefficace. Il a été décidé 
de retirer le piercing lingual. Les symptômes ont alors disparu en 48 heures et n'étaient pas réapparus un an plus tard. Il n'y avait pas de lésion du trijumeau mais probablement une irritation chimique ou galvanique de ce dernier.

\section{Complications odontologiques}

Après la phase de cicatrisation, les complications les plus fréquentes sont odontologiques. Sur les dents, on peut observer des fêlures, des fractures avec ou sans atteinte pulpaire, des abrasions... Les dents peuvent aussi endurer un phénomène dit de burinage par les bijoux ventraux ou dorsaux [72] Il en résulte à terme des symptômes tels que la sensibilité au froid, au sucre, des douleurs à la pression, etc. La littérature est extrêmement riche en cas cliniques concernant des fractures dentaires consécutives au port de piercing [26,55, 7377]. Si la perte de substance est trop importante, on peut être conduit à extraire la dent. De Moor et al. ont présenté trois cas de délabrements dentaires dus à des piercings linguaux, deux de ces cas se sont soldés par la perte de la dent endommagée [18]. Les mêmes auteurs ont rapporté que les éléments prothétiques, en céramique particulièrement, ne sont pas non plus à l'abri de fractures. Le perçage de la langue est fortement lié à l'ébréchure, la fracture et la fissure des dents et à l'usure du bord incisif. Selon De Moor et al., 80 \% des patients avec un piercing de la langue présentent une perte de substance dentaire et une ébréchure des couronnes en céramique [78]. Il y a de plus une corrélation positive entre la longueur des tiges de type barbell utilisées dans le piercing de la langue et les dommages faits aux tissus durs. Campbell et al. affirment que $47 \%$ des individus porteurs de piercings linguaux présentent des pertes de substance dentaire au bout de 4 ans et plus de port d'un piercing [79]. Le port à long terme d'un piercing lingual est directement corrélé à une augmentation du risque de fracture dentaire.

Lors d'une anesthésie à l'épine de Spix, le patient peut être incapable de contrôler la langue anesthésiée. Si le patient est porteur d'un piercing, le risque de mordre involontairement le piercing est important.

Des migrations dentaires peuvent également être constatées si le patient prend l'habitude de pousser le bijou entre les dents. Dans les deux cas décrits par Bethke et Reichart, des diastèmes sont apparus sur une période de 3 à 4 ans au point de permettre à ces patients de faire passer toute la barre entre les incisives [80].

Sur le parodonte, on peut observer des inflammations et des œdèmes de la gencive [55], des récessions [81], des pertes d'attache [82]... Ces atteintes parodontales en rapport avec les piercings linguaux sont très bien documentées [8386]. Comme pour celles des tissus dentaires, l'atteinte parodontale peut mettre en péril la dent. Zadik et al. rapportent le cas d'une jeune fille de 18 ans ayant une mobilité importante des incisives mandibulaires liée à une perte osseuse conséquente en rapport avec le port d'un piercing lingual. L'atteinte des tissus parodontaux des dents des secteurs antérieurs sont fréquentes chez les porteurs de piercing lingual, intéressant aussi bien la gencive $[79,87]$ que l'os $[88,89]$. Ainsi des récessions linguales sur les incisives centrales mandibulaires sont retrouvées chez $50 \%$ des individus porteurs de barbell de plus de $16 \mathrm{~mm}$ depuis 2 ans ou plus [79].

Ces lésions sont souvent dues à des parafonctions, le patient va par exemple «jouer » avec son piercing en le faisant rouler contre la gencive linguale et palatine notamment dans le secteur antérieur [90] où il a tendance à serrer l'extrémité dorsale entre les dents [79].

Au total, les piercings linguaux sont associés à une morbidité odontologique significative à court terme et à long terme [91].

\section{Formation des praticiens et information des patients}

Il est indispensable que les professionnels de santé disposent des informations et des connaissances nécessaires à la prise en charge des patients souhaitant se faire percer (facteurs de risques, complications éventuelles, délais de cicatrisation, conseils d'hygiène...) mais également des individus déjà percés qui présentent des pathologies en relation avec leur piercing (indications de dépose du bijou, manipulations du bijou, etc.) [92]. À titre d'exemple, une enquête menée auprès des infirmières scolaires de Franche Comté pendant l'automne 2004 indiquait que $78 \%$ d'entre elles avaient déjà été sollicitées pour un problème de piercing. Il est certain que l'augmentation du nombre d'individus percés se traduira forcément par une augmentation des consultations médicales et dentaires en rapport avec les piercings linguaux [93]. L'information du personnel de santé est d'autant plus importante qu'il est fréquent que les candidats au piercing n'ont aucune conscience des risques liés à ce type d'acte. Levin et al. ont relaté que chez 389 patients avec des piercings, 225 (57,8\%) ignoraient les dangers du piercing intrabuccal [85].

Il serait souhaitable que tous les cabinets dentaires et médicaux offrent des dépliants ou toute autre documentation permettant aux patients de mieux être informés au sujet du piercing buccal et de ses complications [94].

\section{Manipulation du piercing}

Lorsque certaines complications surviennent ou tout simplement pour certains examens d'imagerie médicale, il peut s'avérer nécessaire de retirer le piercing. Si le médecin ou le chirurgien dentiste sont familiarisés avec le mécanisme d'ouverture, ceci ne posera aucun souci. Or une étude réalisée dans des services d'urgence a montré que moins d'un médecin urgentiste sur quatre était capable de décrire précisément le mécanisme d'ouverture du piercing le plus commun [27]. Comme les complications surviennent souvent chez des patients eux-mêmes peu expérimentés en la matière, il convient de former le personnel à la manipulation des piercings (clip ou vis), en particulier dans les services d'urgence hospitaliers, 
afin que l'ablation par section à la pince, potentiellement responsable de dégâts tissulaires (les alliages habituels sont très coupants après cisaillement à la pince), soit proscrite.

Il est intéressant de noter qu'un « kit de retrait de piercing dans les situations d'urgence » [95] existe aux États-Unis : ce kit, accompagné de photos, permet aux praticiens de se familiariser avec les différentes techniques de retrait de bijoux mais aussi avec les techniques de remplacement provisoire du piercing à des fins de maintien de l'orifice durant un acte opératoire par exemple $[96,97]$. Pour Petitcolas et al., les cabinets dentaires et les services hospitaliers devraient avoir à disposition des bijoux non métalliques temporaires, à mettre en place durant certains actes médicaux notamment chirurgicaux, puis retirés [90]. On pourra par exemple utiliser du nylon.

Lorsqu'une infection survient sur le site de perçage, il est primordial pour le praticien de savoir que le fait de retirer le piercing peut « piéger » l'infection en la privant de la voie de drainage que constitue l'orifice [30]. Il est recommandé dans ces situations de laisser le bijou en place ou de le remplacer par du matériel stérile ou un drain chirurgical. Ce drainage permet d'éviter la formation d'un abcès. Il est indispensable d'informer les patients de la nécessité de conserver le piercing en place jusqu'à ce que le médecin ou le chirurgien dentiste lui dise qu'il peut le retirer. Le retrait du piercing ne doit jamais être fait en première intention [98].

La question de la dépose ou non du piercing lingual avant une intervention chirurgicale ou une anesthésie ne fait pas l'objet d'un consensus. Certains praticiens ne voient aucun inconvénient à laisser en place le piercing [99-102] alors que d'autres justifient en s'appuyant sur des cas cliniques (blessures lors de l'intubation, saignements, difficultés à intuber...) la dépose systématique du piercing lingual [103-105]. D'un côté, certains anesthésistes pensent que si les patients peuvent boire, manger, parler et dormir avec leur piercing, ils peuvent aussi être intubés avec. D'un autre côté, la dépose entraîne de fait l'élimination du risque d'inhalation du bijou et de saignement. Au-delà de la diminution du risque médico-légal, il s'agit pour ces praticiens d'une question de sécurité. Une difficulté liée à l'intervention chirurgicale est que, si l'on veut déposer le piercing, un système de remplacement doit être mis en place pendant l'anesthésie sous peine de voir l'orifice éventuellement se refermer après 2 heures environ [106].

Quoi qu'il en soit, même si le praticien ne dépose pas systématiquement les piercings linguaux lors de l'intubation, il doit avoir les connaissances suffisantes pour le faire, au cas où, en toute sécurité.

\section{Recommandations pré-opératoires}

Lorsqu'un patient évoque au praticien son intention de se faire percer la langue, le médecin ou le chirurgien dentiste doit l'informer de l'ensemble des risques qu'il encourt et qui ont été détaillés précédemment. Il faut rechercher les contre-indications au geste. Le patient doit être interrogé sur d'éventuels problèmes médicaux : recherche d'allergie (iode, antiseptique, métaux, latex...), de prise de médicaments (traitements antérieurs ou en cours, notamment la prise d'anti-inflammatoires), antécédents d'intervention chirurgicale, de diabète, de maladie de peau chronique... [28]. Il importe de lui expliquer que ceux-ci peuvent constituer des contre-indications à la pratique d'un geste de piercing. La présence d'une infection dans la cavité buccale contre-indique la mise en place d'un piercing lingual; ceci signifie que l'état bucco-dentaire doit être correct.

\section{Recommandations post-opératoires}

Le praticien doit être à même de répondre à l'ensemble des questions que peut se poser son patient, que ce soit en terme de délais de cicatrisation, d'évolution normale ou anormale de la cicatrisation, de précautions d'usage...

Les délais de cicatrisation déjà évoqués (3 à 6 semaines) doivent être considérés comme une moyenne, des variations peuvent exister en fonction du « patient » et du type de piercing [107]. Le site du perçage cicatrise de l'extérieur vers l'intérieur, les «patients » peuvent de ce fait croire que le site est parfaitement cicatrisé alors qu'il ne l'est pas complètement : ils arrêtent alors les soins post-opératoires majorant ainsi le risque de complications. Durant la phase initiale de cicatrisation, il s'écoule normalement un liquide jaunâtre du site de perçage; il est constitué de lymphe et de cellules mortes [107]. Une phase d'adaptation au port d'un piercing lingual peut s'avérer nécessaire. Certains auteurs recommandent pendant cette période «d'acclimatation » de déposer la nourriture avec des doigts propres ou des couverts propres, directement sur les molaires et de commencer à mâcher doucement pour éviter de mordre sur le piercing [107].

Afin de minorer le risque de survenue de complications infectieuses, il faut éviter de mettre dans la cavité buccale des objets souillés (mains non lavées, stylos...) ou de partager des couverts avec d'autres personnes. Il est évident qu'une hygiène irréprochable des mains est primordiale lors de la manipulation du bijou. Sucer de la glace, manger et boire des aliments froids peuvent limiter l'œdème lié au perçage. Il est également recommandé de faire pendant au moins 8 à 14 jours des bains de bouche avec une solution antiseptique deux à trois fois par jour (type Eludril ${ }^{\circledR}$, Hextril ${ }^{\circledR}$ ou Givalex ${ }^{\circledR}$ ) notamment après chaque repas pour éviter toute stagnation de débris alimentaires autour du piercing. Ces bains de bouche doivent être débutés le jour même de la pose. Il faut éviter de parler, de fumer, de boire de l'alcool et de manger des aliments épicés ou acides. Suite à la pose d'un piercing lingual il faut également éviter pendant au moins 3 semaines les contacts oral-oral et oral-génital. Un contrôle doit être effectué par un professionnel à $\mathrm{J}+10$. Il est normal d'observer une réaction inflammatoire (érythème et œdème) dans les jours qui suivent la pose d'un piercing. Toutefois ces symptômes doivent disparaître en moins d'une semaine. Aucun traitement médicamenteux, même lorsqu'il s'agit de topiques locaux en vente libre, ne doit être préconisé par l'opérateur. 
Lorsque le site de perçage est totalement cicatrisé, certains praticiens recommandent de retirer le piercing durant les repas pour éviter de mordre par inadvertance sur le piercing.

Lors d'une consultation au cabinet dentaire, même si le piercing n'est pas le motif de consultation, il est recommandé d'en profiter pour vérifier la tenue du bijou et pour nettoyer le piercing ainsi que le site de perçage avec la seringue air/eau, tout en conseillant au patient de retirer régulièrement le piercing pour le brosser. Par contre, il faut que le patient garde à l'esprit qu'il doit immédiatement remettre en place son piercing sous peine de voir le site de perçage se refermer, ce qui peut survenir à partir de la $2^{\text {ème }}$ heure. C'est pour cette raison que le piercing lingual doit être porté au cours du sommeil.

\section{Positions étatiques, scientifiques et militaires}

L'augmentation fulgurante du nombre d'officines de piercing et d'actes de piercing, notamment en France, au cours des dernières décennies ne doit pas submerger les sociétés savantes civiles ni les institutions militaires. L'ensemble des professionnels de santé doit réagir face à ce qui est clairement un problème de santé publique.

\section{Politiques étatiques [108]}

Depuis une dizaine d'années, plusieurs pays à travers le monde ont adopté des mesures, le plus souvent noncoercitives, visant à accompagner les perceurs et les percés de manière à ce que les actes soient pratiqués dans les meilleures conditions possibles, notamment pour l'hygiène, afin de limiter essentiellement le risque infectieux. Des formations sont ainsi proposées aux perceurs en Espagne et en Italie, ce qui n'est pas le cas en Suisse alors qu'en 2005, 400 studios de piercing avaient déjà pignons sur rue (ce qui est considérable comparé au nombre d'habitants). En Italie, la vaccination du personnel des officines de piercing contre l'hépatite $B$ et le tétanos est obligatoire.

Des mesures très contraignantes ont été mises en place dans certains états des États-Unis : le contrôle des officines de piercing est draconien, les obligations en matière d'hygiène comparables à celles imposées aux structures médicales hospitalières. Le résultat a été une diminution du nombre d'officines en raison du coût des installations ou de l'impossibilité de mise aux normes. Les officines restantes ont augmenté leurs tarifs, ce qui s'est traduit par une baisse de la fréquentation des studios de piercing. Par contre le nombre d'actes de piercing n'a pas diminué, les clients se reportant vers des perceurs "sauvages " avec comme risque une augmentation des infections transmises lors du perçage. Le résultat était donc à l'opposé de l'objectif initial. Dans le code belge de bonnes pratiques en matière de piercing, il est indiqué qu'on « ne peut pas médicaliser et réglementer à l'extrême. Il est vrai que si l'on veut atteindre le risque zéro, il vaut mieux proscrire tout piercing et tout tatouage. C'est infaisable ».
Dans ce contexte, la France a adopté une position intermédiaire avec la parution au journal officiel courant février 2008 d'un décret réglementant les activités de perçage et de tatouage [109]. Ce décret institue notamment des conditions d'hygiène pour les locaux, les équipements et les matériels utilisés dans ces pratiques, mais aussi des obligations en matière d'information du consommateur. Les personnes mettant en œuvre ces techniques sont tenues par une obligation d'information des clients quant aux risques et précautions à respecter (article R. 1311-12 du code de la santé publique). Cette information doit être affichée dans le local et remise par écrit aux clients. Elles doivent se déclarer auprès du préfet de département du lieu d'exercice de leur activité (article R1311-1). Elles doivent également avoir suivi une formation aux conditions d'hygiène et de salubrité (article R1311-4 du CSP).

L'ensemble de ces politiques est en adéquation avec la gestion du risque infectieux; mais comme on l'a vu ci-dessus, de nombreuses complications sont inhérentes à la pose et au port du piercing indépendamment de la technique de pose utilisée, de la formation du perceur ou de l'asepsie des locaux. L'ensemble de la problématique n'est absolument pas prise en compte actuellement par les États.

\section{Recommandations civiles}

Afin de comprendre l'impact médical du piercing lingual, il suffit de voir la diversité des revues dont émanent les articles de la bibliographie : revues d'anesthésie, de chirurgie maxillo-faciale, de chirurgie plastique, de dermatologie, de neurologie, d'infectiologie, de bactériologie, d'urgences médicales, d'odontologie, d'oto-rhino-laryngologie... Ceci doit faire prendre conscience qu'appréhender les complications liées aux piercings linguaux sous le seul angle infectieux est réducteur et surtout erroné.

Du fait de ces complications délétères, certaines sociétés savantes civiles se sont montrées beaucoup plus fermes que les politiques étatiques. L'ADA (American Dental Association) s'est ainsi prononcé clairement contre les piercings linguaux [110]. L'AAPD (American Academy of Pediatric Dentistry) s'est dite fortement opposée aux piercings intra-buccaux et péri-buccaux dès 2000, puis a réaffirmé sa position en 2003 et en 2007. L'AAPD justifie sa position par le risque considérable que représente le piercing pour les tissus intrabuccaux et péri-buccaux. Cette prise de position s'appuie sur une méta-analyse des complications liées aux piercings buccaux réalisée à partir de Medline [111]. La BDA (British Dental Association), le NIH (National Institute of Health), le NHF (National Hepatitis Fondation) et beaucoup d'autres organisations professionnelles affirment que le piercing lingual engendre un risque inacceptable pour la santé et que le meilleur conseil que puisse donner un dentiste est de ne pas se faire percer.

On a donc d'un côté la «science » qui affirme qu'il faut impérativement arrêter les piercings de la langue et de l'autre les «États » qui cherchent à ce que ces pratiques se fassent 
dans les meilleurs conditions possibles. Il est certain qu'à partir du moment où ces actes de perçage sont légaux, on doit envisager les éventuelles complications. Les professionnels de santé, quelle que soit leur opinion, se doivent donc d'accompagner leurs patients afin de minorer les risques de complications

\section{Règlements militaires}

Trois armées au moins ont fait le choix de l'interdiction totale des piercings linguaux.

L'armée américaine, dans le chapitre 1-14 de son texte de référence sur la tenue et l'apparence des uniformes militaires et des insignes [112], s'intéresse plus particulièrement au port de bijou; ;'alinéa c. porte sur le « body piercing ». Il y est écrit que lorsque le militaire se trouve dans une enceinte militaire ou dépendante de l'armée, il ne doit pas porter de bijoux, objets, ornementations... attachés ou fixés sur ou à travers la peau, qu'il soit en uniforme ou en civil, en service ou non. Le terme de peau est bien précisé : il ne s'agit pas uniquement de la peau " extérieure », mais aussi de la langue, des lèvres, de la cavité buccale et des autres surfaces corporelles non visibles.

L'armée israélienne a également opté pour l'interdiction, après le décès, en septembre 2008, d'un soldat au Rambam Medical Center d'Haïfa de complications liées à la pose d'un piercing lingual [113]. Il faut noté que le pourcentage d'adolescent porteur d'un piercing lingual est deux fois plus important en Israël qu'à New-York. Ce cas de décès lié à un piercing lingual a entraîné le bannissement de cette pratique au sein de l'ensemble des forces armées israéliennes [114].

L'armée australienne décourage en général la pratique du piercing [115]. Que ce soit au sein de l'armée de terre, de l'armée de l'air ou de la marine, le port de tout piercing (visible ou non) est interdit durant le service mais aussi en dehors du service tant que le militaire se trouve dans une enceinte militaire [116].

Ceci montre bien que des positions tranchées sont possibles et qu'elles sont applicables et appliquées.

\section{Discussion}

Le piercing lingual est une réalité au sein de la société française et bien entendu de l'armée. Cette pratique n'étant d'ailleurs pas interdite au sein de l'armée, elle est de fait acceptée. Aucune donnée épidémiologique militaire n'est actuellement disponible pour la simple raison que le personnel porteur de tels bijoux n'est pas systématiquement recensé. À titre personnel, lors de l'examen bucco-dentaire réalisé dans le cadre des visites systématiques annuelles ou avant projection sur des théâtres d'opération, on recense la présence de piercings linguaux. L'analyse d'une base de données de plus de 20000 odontogrammes de militaires français examinés entre 2006 et 2008 [117] fait ressortir une grande disparité non pas entre les types d'unité ou d'armée mais en fonction des compagnies, des batteries... auxquelles appartient le militaire. Il apparaît clairement dans le cadre militaire un phénomène de groupe : au sein d'une unité élémentaire, soit personne ou presque n'a de piercing lingual, soit un grand nombre de militaires en possèdent un (jusqu'à près de $35 \%$ dans certaines compagnies), la cible privilégiée étant la population jeune (18-20 ans).

Si l'on se place sur le plan opérationnel, quelles peuvent être les conséquences de ces piercings? Les complications liées à la pose du piercing sont fréquentes avec une infection secondaire à la pose du bijou dans 10 à $30 \%$ des cas. Ces infections et autres complications précédemment décrites peuvent entraîner une indisponibilité du militaire qui devra consulter un médecin, une diminution des performances de l'individu..., mais ceci se déroule logiquement en métropole. Comme on peut espérer que les militaires ne se font pas percer durant une opération extérieure, de ce fait les complications de cette phase initiale devraient se limiter au territoire métropolitain : ceci en facilite la prise en charge et en limite les conséquences opérationnelles.

En fait, le plus important se révèle être les complications liées au port du piercing lingual au cours d'une opération extérieure. On a vu que des fractures dentaires surviennent en chez $50 \%$ des patients ayant un piercing. Ces fractures peuvent :

- être gênantes et blessantes pour la langue du fait de la présence d'un angle vif sur la dent fracturée;

- entraîner une sensibilité au froid, au sucré... secondaire à la perte de tissu dentaire et à la proximité de la pulpe dentaire ;

- être extrêmement douloureuse en cas d'atteinte pulpaire.

De plus, même si cela ne fait pas l'objet d'un consensus, la présence d'un piercing lingual peut compliquer, voire compromettre l'intubation du patient (obstacle, blessure, saignement...) dans des situations d'urgence pouvant survenir sur le théâtre d'opérations extérieures. D'autres complications peuvent également survenir : inhalation du bijou, infections secondaires...

Au total, le piercing lingual représente un facteur de risque réel de diminution de la capacité opérationnelle des forces. La participation de militaires à des opérations de haute intensité (Afghanistan) semble totalement incompatible avec une prise de risque volontaire et non justifiée, à savoir le port d'un piercing lingual. Selon nous et sur la base de la littérature internationale, cette pratique devrait être proscrite au sein des armées au moins sur le théâtre d'opérations extérieures. Il ne s'agit pas de porter un quelconque jugement moral sur les porteurs de piercing, ceci ne concerne que les piercings linguaux dont on a étudié les complications associées et l'impact opérationnel. Cependant, quelle que soit la position de l'armée vis-à-vis du piercing, il semble indispensable aux praticiens du service de santé des armées de se familiariser avec cette pratique de manière à pouvoir conseiller les patients, à minorer les risques de survenue de complications et à gérer les complications lorsqu'elles apparaissent. 


\section{Conclusion}

Le piercing lingual connait un engouement considérable depuis quelques années. Les complications liées à ce type d'acte sont multiples et fréquentes (infections secondaires dans 10 à $30 \%$ des cas, fractures dentaires dans $47 \%$ des cas...). Il s'agit donc d'un véritable problème de santé publique. Or si le risque infectieux est pris en compte par certains États dont la France, seules les sociétés savantes civiles ont su prendre en considération l'ensemble des complications liées à la pose, puis au port du piercing de la langue en recommandant fortement la proscription de ce type d'acte.

L'armée, de part la spécificité du métier de militaire notamment lors des opérations extérieures, ne peut se tenir à l'écart du problème du piercing lingual dont les complications fréquentes, plus particulièrement odontologiques, peuvent diminuer la capacité opérationnelle des forces armées. L'engagement de la France dans des missions de haute intensité comme en Afghanistan devrait amener à interdire, au moins au cours des missions, le port de piercings linguaux.

En attendant, il apparait indispensable que les praticiens, notamment militaires, acquièrent une connaissance suffisante sur les piercings linguaux de manière à prendre en charge les patients porteurs de tels bijoux mais également et surtout à les informer des risques qu'ils encourent pour leur santé en général.

\section{Références}

1. Guiard-Schmid, Picard H, Slama L, Maslo C, Amiel C, Pialux G, Lebrette $M g$, Rozenbaum W. Le piercing et ses complications infectieuses. Un enjeu de santé publique en France. Presse Med 2000;29:1948-56.

2. Wright JW. Modifying the body: piercing and tattoos. Nurs Stand 1995;10:27-30.

3. Muldoon KA. Body piercing in adolescents. J Pediatr Health Care 1997;11:298-301.

4. Association of professional piercers. The Point 1998;1-6:11.

5. Juno A, Vale V. Research No. 12: modern primitives: an investigation of contemporary adornment and ritual. San Francisco Calif RE/search publications; 1989.

6. Tweeten SSM, Rickman LS. Infectious complications of body piercing. Clin Infect Dis 1998;26:735-40.

7. Hendricks WM. Complications of ear piercing: treatment and prevention. Cutis 1991;48:386-94.

8. Loup PJ, Mombelli A. La cavité buccale: nouvelle cible du «piercing ». Rev Mensuelle Suisse Odontostomatol 2002;112:475-82.

9. Reichl RB, Dailey JC. Intraoral body piercing: a case report. Gen Dent 1996;44:346-7.

10. Armstrong ML, Ekmark E, Brooks B. Body piercing: promoting informed decision making. J Sch Nurs 1995;11:20-5.

11. Boardman R, Smith RA. Dental implications of oral piercing. Oral Health 1997;87:23-31.

12. Brossaud S. Tatouage, piercing et risque d'hépatite $C$ : étude prospective et descriptive au centre de dépistage anonyme et gratuit de Nantes. Thèse de doctorat en Médecine, Faculté de Nantes, 2005.
13. Vault Inc. 2007. Vault employee tattoo and body piercing survey. Accessible sur www.hawaii.edu/hivandaids/Vault Employee Tattoo and Body Piercing Survey, 2007. pdf le 19/06/09.

14. Botchway C, Kuc I. Tongue piercing and associated tooth fracture. J Can Dent Assoc 1998;64:803-5.

15. Stirn A. Piercing, perspectives psychosociales d'un phénomène de société. Paediatrica 2003;14:33-7.

16. Farah CS, Harmon DM. Tongue piercing: case report and review of current practice. Austr Dent J 1998;43:386-9.

17. Armstrong ML. Body piercing: what practicing RNs should know. Texas Nurs 1996;70:8-10.

18. De Moor RJ, De Witte AM, De Bruyne MA. Tongue piercing and associated oral and dental complications. Endod Dent Traumatol 2000;16:232-7.

19. Ferguson H, Ward HJ. Reader survey. Body Art 1994 ;20 :24-6.

20. Scully $\mathrm{C}$, Chen M. Tongue piercing (oral body art). Br J Oral Maxillofac Surg 1994;32:37-8.

21. Shinohara EH, Horikawa FK, Ruiz MM, Shinohara MT. Tongue piercing: case report of a local complication. J Contemp Dent Pract 2007;183-9.

22. Biber JT. Oral piercing: the hole story. Northwest Dent 2003;82:13-7.

23. Wolfe FD. The perils of oral piercing. RDH 2006;26:50-57,93.

24. Reichl RB, Dailey JC. Intraoral body-piercing: a case report. Gen Dent 1996;44:346-7.

25. Levin L, Zadik Y, Beker T. Oral and dental complications of intra-oral piercing. Dent Traumatol 2005;21:341-3.

26. Armstrong ML. You pierced what? Pediatr Nurs 1996;22:236-8

27. Khanna R, Kumar SS, Raju BS. Body piercing in the accident and emergency department. J Accid Emerg Med 1999;16: 418-21.

28. Groupe français d'étude et de recherche sur le piercing. Guide des bonnes pratiques sur le piercing. Guide technique à l'usage des professionnels du piercing, recommandations pour la prévention de la transmission des maladies infectieuses. source AP-HP mars 2001.

29. Braithwaite RL. Risks associated with tattooing and piercing. J Public Health Policy 1999;20:459-70.

30. Yagoda Shagam J. Body piercing safety. Health smart, quarterly health newsletter - university of New Mexico sciences center 2006;16:1-4.

31. Rapport de la Commission européenne en charge de la sécurité sanitaire. IP/03/1033 du 17 juillet 2003

32. Akhondi H, Rahimi AR. Haemophilus aphrophilus endocarditis after tongue piercing. Emerg Infect Dis 2002;8:850-1.

33. Tronel $H$, Chaudemanche $H$, Pechier N, Doutrelant L, Hoen B. Endocarditis due to Nesseria mucosa after tongue piercing. Clin Microbiol Infect 2001;7:275-6.

34. Lick S, Edozie S, Woodside K, Conti V. Streptococcus viridans endocarditis from tongue piercing. J Emerg Med 2005;29:57-9.

35. Gazzeri R, Galarza M. Atypical trigeminal neuralgia associated with tongue piercing. J Am Med Assoc 2006;296:1840-2.

36. Folz BJ, Lippert BM, Kuelkens C, Werner JA. Hazards of piercing and facial body art: a report of three patients and literature review. Ann Plast Surg 2000;45:374-81.

37. Huber M, Moore WS, Terezhalmy GT. Oral/perioral piercing. Quintessence Inter 2003;34:722-3.

38. Perkins CS, Meismer J, Harrison JM. A complication of tongue piercing. Br Dent J 1997;182:147-8. 
39. Murray T. Report of brain abscess wasn't tongue-in-check. Medical Post [online]. 2001;37.

40. Martinello RA, Cooney EL. Cerebellar brain abscess associated with tongue piercing. Clin Infect Dis 2003;36:32-4.

41. Dyce 0, Bruno JR, Hong D, Silverstein K, Brown MJ, Mirza N. Tongue piercing: the new "rusty nail"? Head Neck 2000;22:728-32.

42. Kaldor JM, Archer GT, Buring ML, Ismay SL, Kenrick KG, Lien AS. Risk factor for hepatitis C virus infection in blood donors: a case control study. Med J Aust 1992:157:227-30.

43. Maclennan S, Moore MC, Hewitt PE, Nicholas S, Barbara JJA. A study of anti-hepatitis $C$ positive blood donors: the first year of screening. Transfusion Med 1994;4:125-33.

44. Brandao-Ajacio BM, Fuchs SC. Risks factors for hepatitis C virus infection among blood donors in southern Brazil: a case control study. BMC Gastroenterol 2002;2:18.

45. Recommandations du conseil concernant l'admissibilité des donneurs de sang et de plasma. Journal officiel des communautés européennes 1998;L203/14; L 230/22.

46. Theodossy T. A complication of tongue piercing. A case report and a review of literature. Br Dent J 2003;194:551-2.

47. Pugatch D, Mileno M, Rich JD. Possible transmission of human immunodeficiency virus type 1 from body piercing. Clin Infect Dis 1999;26:767-8.

48. Lakhan SE, Harle L. Fatal fulminant herpes simplex hepatitis secondary to tongue piercing in an immunocompetent adult: a case report. J Med Case Reports 2008;2:356.

49. Farah CS, Harmon DM. Tongue piercing: case report and review of current practice. Aust Dent J 1998;43:387-9.

50. Hardee $P$, Mallya $L$, Hutchinson I. Tongue piercing resulting in hypotensive collapse. Br Dent J 2000;188:657-8.

51. Keogh IJ, O'leary G. Serious complication of tongue piercing. J Laryngol Otol 2001;115:233-4.

52. Shinohara EH, Horikawa FK, Ruiz MM, Shinohara MT. Tongue piercing: case report of a local complication. J Contemp Dent Pract 2007;8:1-4.

53. Lopez-Jornet P, Camacho-Alonso F, Pons-Fuster JM. A complication of lingual piercing: a case report. Oral Surg Oral Med Oral Pathol Oral Radiol Endod 2005;99:18-9.

54. Rosivack G, Kao JY. Prolonged bleeding following tongue piercing: a case report and a review of complications. Paediatr Dentist 2003;25:154-6.

55. Boardman R, Smith RA. Dental implications of oral piercing. J Calif Dent Assoc 1997;25:207.

56. Fleming PS, Flood TR. Bifid tongue: a complication of tongue piercing. Br Dent J 2005;198:265-6.

57. Botchway C. Le besoin de normaliser le perçage de la langue. J Can Dent Assoc 2001;67:18-9.

58. Shteyer A, Howell RM. Tissue reactions to chronic irritation of the tongue in germ-free and conventional rats. J Oral Surg 1970;28:109-12.

59. Keogh IJ, O'leary G. Serious complication of tongue piercing. J Laryngol 0tol 2001;115:233-4.

60. Stirn A. Body piercing: medical consequences and psychological motivations. Lancet 2003;361:1205-15.

61. Reyna GC, Gomez FA, Sanchez RA. Broncoaspiracion de cuerpo extrano por «piercing » lingual. Rev Inst Nal Enf Resp Mex 2008;21:26-8.

62. Neiberger E. A large hypertrophic-keloid lesion associated with tongue piercing: a case report. Gen Dent 2006;54:46-7.
63. $\mathrm{Ng} \mathrm{KH}$, Siar CH, Ganesapillai T. Sarcoid-like foreign body reaction in body piercing: a report of two cases. Oral Surg Oral Med Oral Pathol Oral Radiol Endod 1997;84:28-31.

64. De Moor R, De Witte A, De Buyne M. Tongue piercing and associated oral and dental complications. Endod Dent Traumatol 2000;16:232-7.

65. Er N, Ozkavaf A, Berberoglu A, Yamalik N. An unusual cause of gingival recession: oral piercing. J Period 2000;71:1767-9.

66. Samantha $S$, Tweeten M, Rickman L. Infectious complications of body piercing. Clin Infect Dis 1998;26:735-40.

67. Koenig L, Carnes M. Body piercing, medical concerns with cutting-edge fashion. J Gen Intern Med 1999;14:379-85.

68. Folz BJ, Lippert BM, Kuelkens C, Werner JA. Hazards of piercing and facial body art: a report of three patients and literature review. Ann Plast Surg 2000;45:374-81.

69. Abramovits W, Stevenson LC. Hand eczema in a 22-yearold woman with piercings. Proc (Bayl Univ Med Cent). 2004;17:211-3.

70. Dron P, Lafourcade MP, Leprince F, Nonotte-Varly C, Van Der Brempt X, Banoun L, Sullerot I, This-Vaissette C, Parisot L, Moneret-Vautrin DA. Les allergies aux piercings et aux tatouages : enquête sur réseau d'allergo-vigilance. $\mathrm{Rev} \mathrm{Fr}$ Allergol Immunol Clin 2007;47:398-401.

71. Ehrlich A, Kucenic M, Belsito DV. Role of body piercing in the induction of metal allergies. Am J Contact Dermatol 2001;12:151-5.

72. Diangelis AJ. The lingual barbell: a new etiology for the cracked-tooth syndrome. J Am Dent Assoc 1997;128:1438-9.

73. Brennan M, O'Connell $P, O^{\prime}$ Sullivan M. Multiple dental fractures following tongue barbell placement: a case report. Dent Traumatol 2006;22:41-3.

74. Croll TP. "Wrecking ball" dental fractures: report of 2 cases. Quintescence Int 1999;30:275-7.

75. Ram D, Peretz B. Tongue piercing and insertion of metal studs: three cases of dental and oral consequences. J Dent Child 2000;67:326-9.

76. Botchway C, Kuc I. Tongue piercing: case report and associated tooth fracture. J Can Dent Assoc 1998;64:803-5.

77. Brennan M, O'Connell B, O'Sullivan M. Multiple dental fractures following tongue barbell placement: a case report. Dent Traumatol 2006;22:41.

78. De Moor RJ De Witte AM, Delme KI, De Bruyne MA, Hommez GM, Goyvaerts D. Dental and oral complications of lip and tongue piercings. Br Dent J 2005;199:506-9.

79. Campbell A, Moore A, Williams E, Stephens J, Tatakis DN. Tongue piercing: impact of time and barbell stem length on lingual gingival recession and tooth chipping. $\mathrm{J}$ Periodontol 2002;73:289-97

80. Bethke G, Reichart PA. Risiken des oralen Piercings. Mund Kiefer Gesichtschir 1999;3:98-101.

81. Dibart S, Defeo P, Surabian G, Hart A, Capri D, Su MF. Oral piercing and gingival recession: review of the literature and a case report. Quintessence Int 2002;33:110-2.

82. Kretchmer MC, Moriarty JD. Metal piercing through the tongue and localized loss of attachment: a case report. J Periodontol 2001;72:831-3.

83. Berenguer G, Forrest A, Horning GM, Towle HJ, Karpinia K. Localized periodontitis as a long term effect of oral piercing: a case report. Compend Contin Educ Dent 2006;27:24-7. 
84. Shacham R, Zaguri A. Tongue piercing and its adverse effects. Oral Surg Oral Med Oral Pathol Oral Radiol Endod 2003;95: 274-6.

85. Levin L, Zadik Y, Becker T. Oral and dental complications of intraoral piercing. Dent Traumatol 2005;21:341-3.

86. Zadik Y, Sandler V. Periodontal attachment loss due to applying force by tongue piercing. J Calif Dent Assoc 2007;35:551-3.

87. Brooks JK, Hooper KA. Formation of mucogingival defects associated with intraoral and perioral piercing: case reports. J Am Dent Assoc 2003;134:837-43.

88. Choe J, Almas K. Tongue piercing as risk factor to periodontal health. NY State Dent J 2005;71:40-3.

89. Panagakos FS, Linfante J. Attachment loss associated with the presence of a tongue bar: a case report. Gen Dent 2000;48: 454-6.

90. Peticolas T, Tilliss T, Cross-Poline G. Oral and perioral piercing: a unique form of self-expression. J Contemp Dent Pract 2000;1:1-10.

91. Stead LR, Williams JV, Williams AC, Robinson CM. An investigation into the practice of tongue piercing in the south west of England. B Dent J 2006;200:103-7.

92. Janssen KM, Cooper BR. Oral piercing: an overview. Internet J Allied Health Sci Pract 2008;6:1-3. http://ijahsp.nova.edu

93. Venta I, Lakoma A. Oral piercings among first-year university students. Oral Surg Oral Med Oral Pathol Oral Radiol Endod 2005;99:546-9.

94. Stringer B. Perçages buccaux et péribuccaux : que puis-je faire, que puis-je dire? J Can Dent Assoc 2007;72:901-2.

95. Amundson TB, Brown JS, Deboer SL. Body piercing stepby-step guide for jewelry removal health care professionals'handbook. Journal of emergency nursing 2006;32:443.

96. Pandit JJ. Potential hazard of radiolucent body art in the tongue. Anesth Analg 2000;91:1564-5.

97. Brown DC. Anaesthetic considerations of a patient with a tongue piercing and a safe solution. Anesthesiology 2000;93:307-8.

98. British Columbia - ministry of health and ministry responsible for seniors guidelines for ear and body piercing. Accessible sur www.llbc.leg.bc.ca/public/pubdocs/356620/PHI082.pdf le 19/06/09.

99. Oyos T. Intubation sequence for patient presenting with tongue ring. Anaesthesiology 1998;88:279.

100. Mandabach M, McCann D, Thompson G. Body art: another concern for the anaesthesiologist. Anaesthesiology 1998;88: 279-80.
101. Rosenberg AD, Young M, Bernstein RL, Albert DB. Tongue rings: just say no. Anaesthesiology 1998;89:1279-80.

102. Girgis Y. Hypoxia caused by body piercing. Anesthesia 2000;55:413.

103. Kuczkowski KM, Benumof JL. Tongue piercing and obstetric anesthesia: is there a cause for concern? J Clin Anesth 2002;14:447-8

104. Wise H. Hypoxia caused by body piercing. Anaesthesia 1999:54:1129.

105. Meltzer DI. Complications of body piercing. Am Fam Physician 2005; 72:2029-34, 35-6.

106. Scully C. Oral piercing in adolescent. CPD Dentistry 2001;2: 79-81.

107. Little LT. Aftercare guidelines for oral piercing. Dental Hygiene News 2005. accessible sur www.ms-flossy.com/piercing.html le 19/06/09.

108. Lafont E. Mémoire pour le diplôme de médecin inspecteur de santé publique. Ecole nationale de la santé publique, 2005.

109. Décret n²008-149 fixant les conditions d'hygiène et de salubrité relatives aux pratiques de tatouage avec effraction cutanée et du perçage, et modifiant le code de la santé publique (disposition réglementaire) paru au Journal Officiel du 20 février 2008.

110. American Dental Association. Current policies, prevention and health education, policy statement on intraoral/perioral piercing, Chicago, American Dental Association 1998;743.

111. AAPD. Policy on intraoral et perioral piercing. Reference manual 2007/2008; 29:54-5.

112. Army Regulation 670-1. Wear and appearance of army uniforms and insigna. Headquarters - department of the Army Wasinghton DC. 3 février 2005.

113. Siegel-Itzkovich J. Soldier's tongue piercing causes fatal infection. Jerusalem Post du 23 septembre 2008.

114. Associated Press. Tongue piercing blamed in soldier's death. Dépêche AP - 22nd of september 2008.

115. Grey LA. Body piercing. From the warrant officer's desk. ADF Health 2006;7:66-7.

116. Army Standing Orders on Dress (ASOD). Australian Army 1, paragraph 3.53, 2002.

117. Gunepin M, Derache F. Impact opérationnel et prise en charge des pathologies bucco-dentaires dans le cadre des opérations extérieures. Méd Armées 2009. Accepté pour publication. 